as important as nerve-sparing in reducing the problem of erectile dysfunction. Although coronal MRI and MRI angiography are useful for research purposes, the authors note that axial MRI images are sufficient for clinical use and they propose that this approach be examined in large, prospective studies.

Original article McLaughlin PW et al. (2005) Vessel-sparing prostate radiotherapy: dose limitation to critical erectile vascular structures (internal pudendal artery and corpus cavernosum) defined by MRI. Int J Radiat Oncol Biol Phys 61: $20-31$

\section{Penile venous surgery in erectile dysfunction}

Penile venous surgery is widely considered to be an outdated and inappropriate treatment for veno-occlusive erectile dysfunction. Having recently reviewed the cases of 21 patients who underwent penile venous stripping or ligation 17 years previously, Chen and colleagues suggest that this practice should be revisited.

Using the abridged, 5-item version of the International Index of Erectile Function (IIEF-5) scoring system, the degree of erectile function was assessed after stripping $(n=16)$ or simple ligation $(n=5)$ of the deep dorsal vein. The preoperative IIEF-5 score was assessed retrospectively. Seventeen years after surgery, the mean IIEF-5 score was unchanged in the ligation group compared with the preoperative score. Patients who underwent venous stripping, however, showed an increase in mean IIEF-5 score, from 9.8 4.1 preoperatively to $18.9 \pm 2.1$ after 17 years. Cavernosograms confirmed these findings; compensatory veins were observed 17 years after surgery in the ligation group, whereas no venous recurrence was evident in the patients who underwent stripping.

Concluding that recurrence of erectile dysfunction following venous surgery might not be due to venous regeneration, the authors suggest that the benefits of this type of surgery might, therefore, be durable. They propose that further studies into the physiology of penile erection and the potential role of venous surgery are warranted.

Original article Chen S-C et al. (2005) The progression of the penile vein: could it be recurrent? J Androl 26: 53-60

\section{Repeat retroperitoneal lymph node dissection for metastatic testicular cancer}

Patients with advanced testicular cancer occasionally require repeat retroperitoneal lymph node dissection (RPLND) due to incomplete resection or recurrence of retroperitoneal masses. Heidenreich et al. have carried out a retrospective analysis of patients undergoing repeat RPLND at their center.

A total of 18 patients with metastatic testicular cancer underwent primary nerve-sparing RPLND $(n=4)$ or residual tumor resection $(n=14)$. All had recurrent or residual retroperitoneal masses (median diameter $7.8 \mathrm{~cm}$ ) and were referred for repeat RPLND. The majority of the recurrences were at the site of the initial retroperitoneal tumor, indicating that primary surgery had been inadequate. Histological examination identified mature teratoma or viable cancer in 10 patients, all of whom received additional chemotherapy. The resected specimens of the remaining eight patients indicated necrosis/fibrosis. The overall disease-specific survival rates after a mean follow-up of 22 months were $85 \%, 50 \%$ and $100 \%$ for patients with mature teratoma, viable cancer or necrosis, respectively.

Heidenreich et al. note that repeat RPLND is technically demanding and so requires thorough preoperative work-up and extensive, interdisciplinary clinical experience. Resection of adjacent organs or vascular structures, for example, is necessary in as many as half of repeat RPLND cases. The authors conclude that the procedure should be carried out only at centers of expertise, where its safety and efficacy can be maximized.

Original article Heidenreich A et al. (2005) Repeat retroperitoneal lymphadenectomy in advanced testicular cancer. Eur Urol 47: 64-71

\section{A novel technique for varicocelectomy}

A recent report from Taiwan describes a new, minimally-invasive procedure for treating scrotal varicoceles. Compared with conventional, laparoscopic varix ligation, this approach appears to decrease postoperative pain, accelerate convalescence and improve cosmetic results. 